Some spaces of difference sequences and lacunary statistical convergence in $n$-normed space defined by sequence of Orlicz functions

M. Mursaleen, Kuldip Raj, and Sunil K. Sharma 


\title{
SOME SPACES OF DIFFERENCE SEQUENCES AND LACUNARY STATISTICAL CONVERGENCE IN $n$-NORMED SPACE DEFINED BY SEQUENCE OF ORLICZ FUNCTIONS
}

\author{
M. MURSALEEN, KULDIP RAJ, AND SUNIL K. SHARMA \\ Received 24 September, 2013
}

\begin{abstract}
In the present paper we introduce some new sequence spaces in the n-normed space. We define here some new spaces of double sequences by using the notions of lacunary sequences, difference sequences, almost convergence, sequence of Orlicz functions and statistical convergence. We examine some topological properties of these spaces of double sequences, inclusion relations between these newly defined sequence spaces and establish relation with lacunary statistical convergence. The study of these new sequence spaces provides a tool to deal with convergence problems of double sequences.
\end{abstract}

2010 Mathematics Subject Classification: 40A05; 46A45; 46B70

Keywords: difference sequences, double sequence spaces, Orlicz function, $n$-normed space, statistical convergence, lacunary statistical convergence

\section{INTRODUCTION AND PRELIMINARIES}

The initial work on double sequences is found in Bromwich [5]. Later on, it was studied by Hardy [14], Moricz [21], Moricz and Rhoades [22], Mursaleen [24], [23], Basarir and Sonalcan [4], Altay and Basar [2], Basar and Sever [3], Mursaleen and Mohiuddine [29,30], Alotaibi et al [1] and many others. Mursaleen and Edely [27] have recently introduced the statistical convergence and Cauchy convergence for double sequences and given the relation between statistical convergent and strongly Cesaro summable double sequences. Nextly, Mursaleen [24] and Mursaleen and Edely [28] have defined the almost strong regularity of matrices for double sequences and applied these matrices to establish a core theorem and introduced the $M$-core for double sequences and determined those four dimensional matrices transforming every bounded double sequences $x=\left(x_{k, l}\right)$ into one whose core is a subset of the $M$ core of $x$. More recently, Altay and Basar [2] have defined the spaces $\mathcal{B} \&, \mathcal{B} \&(t)$,

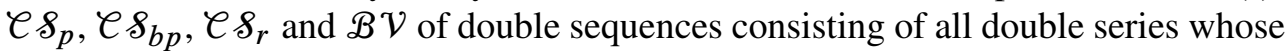
sequence of partial sums are in the spaces $\mathcal{M}_{u}, \mathcal{M}_{u}(t), \mathcal{C}_{p}, \mathcal{C}_{b p}, \mathcal{C}_{r}$ and $\mathscr{L}_{u}$, respectively and also examined some properties of these sequence spaces and determined 


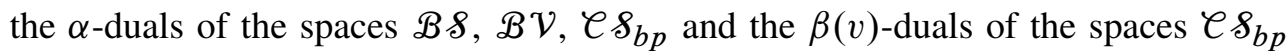
and $\mathcal{C} \delta_{r}$ of double series. Now, recently Basar and Sever [3] have introduced the Banach space $\mathscr{L}_{q}$ of double sequences corresponding to the well known classical sequence space $\ell_{q}$ and examined some properties of the space $\mathscr{L}_{q}$. By the convergence of a double sequence we mean the convergence in the Pringsheim sense i.e. a double sequence $x=\left(x_{k, l}\right)$ has Pringsheim limit $L$ (denoted by $P-\lim x=L$ ) provided that given $\epsilon>0$ there exists $n \in N$ such that $\left|x_{k, l}-L\right|<\epsilon$ whenever $k, l>n$ see [36]. We shall write more briefly as $P$-convergent. The double sequence $x=\left(x_{k, l}\right)$ is bounded if there exists a positive number $M$ such that $\left|x_{k, l}\right|<M$ for all $k$ and $l$.

An Orlicz function $M$ is a function, which is continuous, non-decreasing and convex with $M(0)=0, M(x)>0$ for $x>0$ and $M(x) \longrightarrow \infty$ as $x \longrightarrow \infty$.

Lindenstrauss and Tzafriri [15] used the idea of Orlicz function to define the following sequence space. Let $w$ be the space of all real or complex sequences $x=\left(x_{k}\right)$, then

$$
\ell_{M}=\left\{x \in w: \sum_{k=1}^{\infty} M\left(\frac{\left|x_{k}\right|}{\rho}\right)<\infty\right\}
$$

which is called as an Orlicz sequence space. The space $\ell_{M}$ is a Banach space with the norm

$$
\|x\|=\inf \left\{\rho>0: \sum_{k=1}^{\infty} M\left(\frac{\left|x_{k}\right|}{\rho}\right) \leq 1\right\} .
$$

It is shown in [15] that every Orlicz sequence space $\ell_{M}$ contains a subspace isomorphic to $\ell_{p}(p \geq 1)$. The $\Delta_{2}$-condition is equivalent to $M(L x) \leq k L M(x)$ for all values of $x \geq 0$, and for $L>1$. The notion of difference sequence spaces was introduced by Kızmaz [17], who studied the difference sequence spaces $l_{\infty}(\Delta), c(\Delta)$ and $c_{0}(\Delta)$. The notion was further generalized by Et and Çolak [7] by introducing the spaces $l_{\infty}\left(\Delta^{n}\right), c\left(\Delta^{n}\right)$ and $c_{0}\left(\Delta^{n}\right)$.

Let $n$ be non-negative integers, then for $Z=c, c_{0}$ and $l_{\infty}$, we have sequence spaces

$$
Z\left(\Delta^{n}\right)=\left\{x=\left(x_{k}\right) \in w:\left(\Delta^{n} x_{k}\right) \in Z\right\},
$$

where $\Delta^{n} x=\left(\Delta^{n} x_{k}\right)=\left(\Delta^{n-1} x_{k}-\Delta^{n-1} x_{k}\right)$ and $\Delta^{0} x_{k}=x_{k}$ for all $k \in \mathbb{N}$, which is equivalent to the following binomial representation

$$
\Delta^{n} x_{k}=\sum_{v=0}^{n}(-1)^{v}\left(\begin{array}{c}
n \\
v
\end{array}\right) x_{k+v} .
$$

Taking $n=1$, we get the spaces $l_{\infty}(\Delta), c(\Delta)$ and $c_{0}(\Delta)$ studied by Et and Çolak [7]. The concept of 2-normed spaces was initially developed by Gähler [10] in the mid of 1960 's, while that of $n$-normed spaces one can see in Misiak [20]. Since then, many others have studied this concept and obtained various results, see Gunawan $([11,12])$ and Gunawan and Mashadi [13] and many others. Let $n \in \mathbb{N}$ and $X$ be a linear space over the field $\mathbb{K}$, where $\mathbb{K}$ is field of real or complex numbers of dimension $d$, where 
$d \geq n \geq 2$. A real valued function $\|\cdot, \cdots, \cdot\|$ on $X^{n}$ satisfying the following four conditions:

(1) $\left\|x_{1}, x_{2}, \cdots, x_{n}\right\|=0$ if and only if $x_{1}, x_{2}, \cdots, x_{n}$ are linearly dependent in $X$;

(2) $\left\|x_{1}, x_{2}, \cdots, x_{n}\right\|$ is invariant under permutation;

(3) $\left\|\alpha x_{1}, x_{2}, \cdots, x_{n}\right\|=|\alpha|\left\|x_{1}, x_{2}, \cdots, x_{n}\right\|$ for any $\alpha \in \mathbb{K}$, and

(4) $\left\|x+x^{\prime}, x_{2}, \cdots, x_{n}\right\| \leq\left\|x, x_{2}, \cdots, x_{n}\right\|+\left\|x^{\prime}, x_{2}, \cdots, x_{n}\right\|$

is called a $n$-norm on $X$, and the pair $(X,\|\cdot, \cdots, \cdot\|)$ is called a $n$-normed space over the field $\mathbb{K}$.

For example, we may take $X=\mathbb{R}^{n}$ being equipped with the Euclidean $n$-norm $\left\|x_{1}, x_{2}, \cdots, x_{n}\right\|_{E}=$ the volume of the $n$-dimensional parallelopiped spanned by the vectors $x_{1}, x_{2}, \cdots, x_{n}$ which may be given explicitly by the formula

$$
|| x_{1}, x_{2}, \cdots, x_{n} \|_{E}=\left|\operatorname{det}\left(x_{i j}\right)\right|,
$$

where $x_{i}=\left(x_{i 1}, x_{i 2}, \cdots, x_{i n}\right) \in \mathbb{R}^{n}$ for each $i=1,2, \cdots, n$. Let $(X,\|\cdot, \cdots, \cdot\|)$ be an $n$-normed space of dimension $d \geq n \geq 2$ and $\left\{a_{1}, a_{2}, \cdots, a_{n}\right\}$ be linearly independent set in $X$. Then the following function $\|\cdot, \cdots, \cdot\|_{\infty}$ on $X^{n-1}$ defined by

$$
\left\|x_{1}, x_{2}, \cdots, x_{n-1}\right\|_{\infty}=\max \left\{\left\|x_{1}, x_{2}, \cdots, x_{n-1}, a_{i}\right\|: i=1,2, \cdots, n\right\}
$$

defines an $(n-1)$-norm on $X$ with respect to $\left\{a_{1}, a_{2}, \cdots, a_{n}\right\}$.

A sequence $\left(x_{k}\right)$ in a $n$-normed space $(X,\|\cdot, \cdots, \cdot\|)$ is said to converge to some $L \in X$ if

$$
\lim _{k \rightarrow \infty}\left\|x_{k}-L, z_{1}, \cdots, z_{n-1}\right\|=0 \text { for every } z_{1}, \cdots, z_{n-1} \in X .
$$

A sequence $\left(x_{k}\right)$ in a $n$-normed space $(X,\|\cdot, \cdots, \cdot\|)$ is said to be Cauchy if

$$
\lim _{k, i \rightarrow \infty}\left\|x_{k}-x_{i}, z_{1}, \cdots, z_{n-1}\right\|=0 \text { for every } z_{1}, \cdots, z_{n-1} \in X .
$$

If every Cauchy sequence in $X$ converges to some $L \in X$, then $X$ is said to be complete with respect to the $n$-norm. Any complete $n$-normed space is said to be $n$ Banach space.

A double sequence $x=\left(x_{k, l}\right)$ of real numbers is called almost convergent to a limit $L$ if

$$
P-\lim _{p, q \rightarrow \infty} \sup _{m, n \geq 0}\left|\frac{1}{p q} \sum_{k=m}^{m+p-1} \sum_{l=n}^{n+q-1} x_{k, l}-L\right|=0
$$

i.e. the average value of $\left(x_{k, l}\right)$ taken over any rectangle $\{(k, l): m \leq k \leq m+p-$ $1, n \leq l \leq n+q-1\}$ tends to $L$ as both $p$ and $q$ tends to $\infty$, and this $P$-convergence is uniform in $m$ and $n$.

By a lacunary sequence $\theta=\left(i_{r}\right), r=0,1,2, \cdots$, where $i_{0}=0$, we shall mean an increasing sequence of non-negative integers $h_{r}=\left(i_{r}-i_{r-1}\right) \rightarrow \infty(r \rightarrow \infty)$. The intervals determined by $\theta$ are denoted by $I_{r}=\left(i_{r-1}, i_{r}\right]$ and the ratio $i_{r} / i_{r-1}$ will be 
denoted by $q_{r}$. The space of lacunary strongly convergent sequences $N_{\theta}$ was defined by Freedman [10] as follows:

$$
N_{\theta}=\left\{x=\left(x_{k}\right): \lim _{r \rightarrow \infty} \frac{1}{g_{r}} \sum_{k \in I_{r}}\left|x_{k}-L\right|=0 \text { for some } L\right\} .
$$

The double sequence $\theta_{r, s}=\left\{\left(k_{r}, l_{s}\right)\right\}$ is called double lacunary if there exist two increasing sequences of integers such that

$$
k_{0}=0, h_{r}=k_{r}-k_{r-1} \rightarrow \infty \text { as } r \rightarrow \infty
$$

and

$$
l_{0}=0, \bar{h}_{s}=l_{s}-l_{s-1} \rightarrow \infty \text { as } s \rightarrow \infty .
$$

Let $k_{r, s}=k_{r} l_{s}, h_{r, s}=h_{r} \bar{h}_{s}$ and $\theta_{r, s}$ is determined by $I_{r, s}=\left\{(k, l): k_{r-1}<k \leq\right.$ $k_{r}$ and $\left.l_{s-1}<l \leq l_{s}\right\}, q_{r}=\frac{k_{r}}{k_{r-1}}, \bar{q}_{s}=\frac{l_{s}}{l_{s-1}}$ and $q_{r, s}=q_{r} \bar{q}_{s}$.

Let $\mathcal{M}=\left(M_{k, l}\right)$ be a sequence of Orlicz function and $p=\left(p_{k, l}\right)$ be any factorable double sequence of strictly positive real numbers. Then, we define the following sequence spaces:

$$
\begin{gathered}
{\left[A C_{\theta_{r, s}}, \mathcal{M}, p, \Delta^{v},\|\cdot, \cdots, \cdot\|\right]=\left\{x=\left(x_{k, l}\right) \in w(X):\right.} \\
P-\lim _{r, s} \frac{1}{h_{r, s}} \sum_{(k, l) \in I_{r, s}}\left[M_{k, l}\left(\left\|\frac{\Delta^{v} x_{k+m, l+n}-L}{\rho}, z_{1}, \cdots, z_{n-1}\right\|\right)\right]^{p_{k, l}} \\
=0, \text { uniformly in } m \text { and } n \text { for some } L \text { and } \rho>0\} \\
{\left[A C_{\theta_{r, s}}, \mathcal{M}, p, \Delta^{v},\|\cdot, \cdots, \cdot\|\right]_{0}=\left\{x=\left(x_{k, l}\right) \in w(X):\right.} \\
P-\lim _{r, s} \frac{1}{h_{r, s}} \sum_{(k, l) \in I_{r, s}}\left[M_{k, l}\left(\left\|\frac{\Delta^{v} x_{k+m, l+n}}{\rho}, z_{1}, \cdots, z_{n-1}\right\|\right)\right]^{p_{k, l}} \\
=0, \text { uniformly in } m \text { and } n \text { for some } \rho>0\} .
\end{gathered}
$$

If we take $\mathcal{M}(x)=x$, we have

$$
\begin{aligned}
& {\left[A C_{\theta_{r, s}}, p, \Delta^{v},\|\cdot, \cdots, \cdot\|\right]=\left\{x=\left(x_{k, l}\right) \in w(X):\right.} \\
& P-\lim _{r, s} \frac{1}{h_{r, s}} \sum_{(k, l) \in I_{r, s}}\left[\left(\left\|\frac{\Delta^{v} x_{k+m, l+n}-L}{\rho}, z_{1}, \cdots, z_{n-1}\right\|\right)\right]^{p_{k, l}} \\
& \quad=0, \text { uniformly in } m \text { and } n \text { for some } L \text { and } \rho>0\} \\
& {\left[A C_{\theta_{r, s}}, p, \Delta^{v},\|\cdot, \cdots, \cdot\|\right]_{0}=\left\{x=\left(x_{k, l}\right) \in w(X):\right.}
\end{aligned}
$$




$$
P-\lim _{r, s} \frac{1}{h_{r, s}} \sum_{(k, l) \in I_{r, s}}\left[\left(\left\|\frac{\Delta^{v} x_{k+m, l+n}}{\rho}, z_{1}, \cdots, z_{n-1}\right\|\right)\right]^{p_{k, l}}
$$

$=0$, uniformly in $m$ and $n$ for some $\rho>0\}$.

If we take $p=\left(p_{k, l}\right)=1$, we have

$$
\begin{aligned}
& {\left[A C_{\theta_{r, s}}, \mathcal{M}, \Delta^{v},\|\cdot, \cdots, \cdot\|\right]=\left\{x=\left(x_{k, l}\right) \in w(X):\right.} \\
& P-\lim _{r, s} \frac{1}{h_{r, s}} \sum_{(k, l) \in I_{r, s}}\left[M_{k, l}\left(\left\|\frac{\Delta^{v} x_{k+m, l+n}-L}{\rho}, z_{1}, \cdots, z_{n-1}\right\|\right)\right] \\
& =0, \text { uniformly in } m \text { and } n \text { for some } L \text { and } \rho>0\}
\end{aligned}
$$

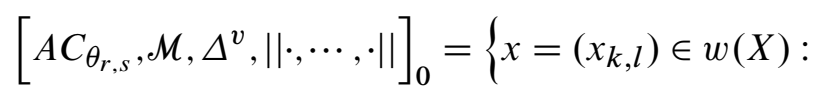

$$
\begin{aligned}
& P-\lim _{r, s} \frac{1}{h_{r, s}} \sum_{(k, l) \in I_{r, s}}\left[M_{k, l}\left(\left\|\frac{\Delta^{v} x_{k+m, l+n}}{\rho}, z_{1}, \cdots, z_{n-1}\right\|\right)\right] \\
& =0 \text {, uniformly in } m \text { and } n \text { for some } \rho>0\} \text {. }
\end{aligned}
$$

If we take $p=\left(p_{k, l}\right)=1$ and $\mathcal{M}(x)=x$, we have

$$
\begin{aligned}
& {\left[A C_{\theta_{r, s}}, \Delta^{v},\|\cdot, \cdots, \cdot\|\right]=\left\{x=\left(x_{k, l}\right) \in w(X):\right.} \\
& P-\lim _{r, s} \frac{1}{h_{r, s}} \sum_{(k, l) \in I_{r, s}}\left(\left\|\frac{\Delta^{v} x_{k+m, l+n}-L}{\rho}, z_{1}, \cdots, z_{n-1}\right\|\right) \\
& =0 \text {, uniformly in } m \text { and } n \text { for some } L \text { and } \rho>0\} \\
& {\left[A C_{\theta_{r, s}}, \Delta^{v},\|\cdot, \cdots, \cdot\|\right]_{0}=\left\{x=\left(x_{k, l}\right) \in w(X):\right.} \\
& P-\lim _{r, s} \frac{1}{h_{r, s}} \sum_{(k, l) \in I_{r, s}}\left(\left\|\frac{\Delta^{v} x_{k+m, l+n}}{\rho}, z_{1}, \cdots, z_{n-1}\right\|\right) \\
& =0 \text {, uniformly in } m \text { and } n \text { for some } \rho>0\} \text {. }
\end{aligned}
$$

The following inequality will be used throughout the paper. If $0 \leq p_{k, l} \leq \sup p_{k, l}=$ $H, D=\max \left(1,2^{H-1}\right)$ then

$$
\left|a_{k, l}+b_{k, l}\right|^{p_{k, l}} \leq D\left\{\left|a_{k, l}\right|^{p_{k, l}}+\left|b_{k, l}\right|^{p_{k, l}}\right\}
$$

for all $k, l$ and $a_{k, l}, b_{k, l} \in \mathbb{C}$. Also $|a|^{p_{k, l}} \leq \max \left(1,|a|^{H}\right)$ for all $a \in \mathbb{C}$. 
The main aim of this paper is to study some topological properties and inclusion relation between the spaces $\left[A C_{\theta_{r, s}}, \mathcal{M}, p, \Delta^{v},\|\cdot, \cdots, \cdot\|\right]$ and $\left[A C_{\theta_{r, s}}, \mathcal{M}, p, \Delta^{v}\right.$, $\|\cdot, \cdots, \cdot\|]_{0}$ in the second section of the paper. In the third section of this paper we defined sequence spaces $\left[\mathcal{F}, \mathcal{M}, p, \Delta^{v},\|\cdot, \cdots, \cdot\|\right]$ and $\left[\mathcal{F}, \mathcal{M}, p, \Delta^{v},\|\cdot, \cdots, \cdot\|\right]$ and proved very interesting inclusion relations between the spaces defined in this section and spaces $\left[A C_{\theta_{r, s}}, \mathcal{M}, p, \Delta^{v},\|\cdot, \cdots, \cdot\|\right]$ and $\left[A C_{\theta_{r, s}}, \mathcal{M}, p, \Delta^{v},\|\cdot, \cdots, \cdot\|\right]_{0}$. We also make an effort to study statistical convergence in the fourth section of this paper.

\section{SOME TOPOLOGICAL PROPERTIES}

Theorem 1. Let $\mathcal{M}=\left(M_{k, l}\right)$ be a sequence of Orlicz functions and $p=\left(p_{k, l}\right)$ be a factorable double sequence of positive real numbers, then the spaces $\left[A C_{\theta_{r, s}}, \mathcal{M}, p, \Delta^{v},\|\cdot, \cdots, \cdot\|\right]$ and $\left[A C_{\theta_{r, s}}, \mathcal{M}, p, \Delta^{v},\|\cdot, \cdots, \cdot\|\right]_{0}$ are linear spaces over the field of complex number $\mathbb{C}$.

Proof. Let $x=\left(x_{k, l}\right), y=\left(y_{k, l}\right) \in\left[A C_{\theta_{r, s}}, \mathcal{M}, p, \Delta^{v},\|\cdot, \cdots, \cdot\|\right]_{0}$ and $\alpha, \beta \in \mathbb{C}$. Then there exist positive numbers $\rho_{1}$ and $\rho_{2}$ such that

$$
\begin{gathered}
\lim _{r, s} \frac{1}{h_{r, s}} \sum_{(k, l) \in I_{r, s}}\left[M_{k, l}\left(\left\|\frac{\Delta^{v} x_{k+m, l+n}}{\rho_{1}}, z_{1}, \cdots, z_{n-1}\right\|\right)\right]^{p_{k, l}}=0, \\
\text { uniformly in } m \text { and } n \text { for some } \rho_{1}>0,
\end{gathered}
$$

and

$$
\lim _{r, s} \frac{1}{h_{r, s}} \sum_{(k, l) \in I_{r, s}}\left[M_{k, l}\left(\left\|\frac{\Delta^{v} x_{k+m, l+n}}{\rho_{2}}, z_{1}, \cdots, z_{n-1}\right\|\right)\right]^{p_{k, l}}=0,
$$

uniformly in $m$ and $n$ for some $\rho_{2}>0$.

Let $\rho_{3}=\max \left(2|\alpha| \rho_{1}, 2|\beta| \rho_{2}\right)$. Since $\mathcal{M}=\left(M_{k, l}\right)$ is non-decreasing convex function, by using inequality (1.1), we have

$$
\begin{aligned}
& \frac{1}{h_{r, s}} \sum_{(k, l) \in I_{r, s}}\left[M_{k, l}\left(\left\|\frac{\Delta^{v}\left(\alpha x_{k+m, l+n}+\beta y_{k+m, l+n}\right)}{\rho_{3}}, z_{1}, \cdots, z_{n-1}\right\|\right)\right]^{p_{k, l}} \\
& \leq D \frac{1}{h_{r, s}} \sum_{(k, l) \in I_{r, s}} \frac{1}{2^{p_{k, l}}}\left[M_{k, l}\left(\left\|\frac{\Delta^{v}\left(x_{k+m, l+n}\right)}{\rho_{1}}, z_{1}, \cdots, z_{n-1}\right\|\right)\right]^{p_{k, l}} \\
& \quad+D \frac{1}{h_{r, s}} \sum_{(k, l) \in I_{r, s}} \frac{1}{2^{p_{k, l}}}\left[M_{k, l}\left(\left\|\frac{\Delta^{v}\left(y_{k+m, l+n}\right)}{\rho_{2}}, z_{1}, \cdots, z_{n-1}\right\|\right)\right]^{p_{k, l}}
\end{aligned}
$$




$$
\begin{aligned}
\leq & D \frac{1}{h_{r, s}} \sum_{(k, l) \in I_{r, s}}\left[M_{k, l}\left(\left\|\frac{\Delta^{v}\left(x_{k+m, l+n}\right)}{\rho_{1}}, z_{1}, \cdots, z_{n-1}\right\|\right)\right]^{p_{k, l}} \\
& +D \frac{1}{h_{r, s}} \sum_{(k, l) \in I_{r, s}}\left[M_{k, l}\left(\left\|\frac{\Delta^{v}\left(y_{k+m, l+n}\right)}{\rho_{2}}, z_{1}, \cdots, z_{n-1}\right\|\right)\right]^{p_{k, l}} \\
\longrightarrow & 0 \text { as } r \longrightarrow \infty, \text { uniformly in } m \text { and } n .
\end{aligned}
$$

Thus, we have $\alpha x+\beta y \in\left[A C_{\theta_{r, s}}, \mathcal{M}, p, \Delta^{v},\|\cdot, \cdots, \cdot\|\right]_{0}$. Hence $\left[A C_{\theta_{r, s}}, \mathcal{M}, p, \Delta^{v}\right.$, $\|\cdot, \cdots, \cdot\|]_{0}$ is a linear space. Similarly, we can prove that $\left[A C_{\theta_{r, s}}, \mathcal{M}, p, \Delta^{v},\|\cdot, \cdots, \cdot\|\right]$ is a linear space.

Theorem 2. For any sequence of Orlicz functions $\mathcal{M}=\left(M_{k, l}\right)$ and $p=\left(p_{k, l}\right)$ be a factorable double sequence of positive real numbers, the space $\left[A C_{\theta_{r, s}}, \mathcal{M}, p, \Delta^{v},\|\cdot, \cdots, \cdot\|\right]_{0}$ is a topological linear space paranormed by $g(x)=$ $\inf \left\{\rho^{\frac{p_{r, s}}{K}}:\left(\frac{1}{h_{r, s}} \sum_{k, l \in I_{r, s}}\left[M_{k, l}\left(\left\|\frac{\Delta^{v} x_{k+m, l+n}}{\rho}, z_{1}, \cdots, z_{n-1}\right\|\right)\right]^{p_{k, l}}\right)^{\frac{1}{K}} \leq 1, r, s \in \mathbb{N}\right\}$, where $K=\max \left(1, \sup _{k, l} p_{k, l}<\infty\right)$.

Proof. Clearly $g(x) \geq 0$ for $x=\left(x_{k, l}\right) \in\left[A C_{\theta_{r, s}}, \mathcal{M}, p, \Delta^{v},\|\cdot, \cdots, \cdot\|\right]_{0}$. Since $M_{k, l}(0)=0$, we get $g(0)=0$. Again, if $g(x)=0$, then $\inf \left\{\rho^{\frac{p_{r, s}}{K}}:\left(\frac{1}{h_{r, s}} \sum_{(k, l) \in I_{r, s}}\left[M_{k, l}\left(\left\|\frac{\Delta^{v} x_{k+m, l+n}}{\rho}, z_{1}, \cdots, z_{n-1}\right\|\right)\right]^{p_{k, l}}\right)^{\frac{1}{K}} \leq 1, r, s \in \mathbb{N}\right\}$ $=0$. This implies that for a given $\epsilon>0$, there exists some $\rho_{\epsilon}\left(0<\rho_{\epsilon}<\epsilon\right)$ such that

$$
\left(\frac{1}{h_{r, s}} \sum_{k, l \in I_{r, s}}\left[M_{k, l}\left(\left\|\frac{\Delta^{v} x_{k+m, l+n}}{\rho_{\epsilon}}, z_{1}, \cdots, z_{n-1}\right\|\right)\right]^{p_{k, l}}\right)^{\frac{1}{K}} \leq 1 .
$$

Thus

$$
\begin{aligned}
& \left(\frac{1}{h_{r, s}} \sum_{(k, l) \in I_{r, s}}\left[M_{k, l}\left(\left\|\frac{\Delta^{v} x_{k+m, l+n}}{\epsilon}, z_{1}, \cdots, z_{n-1}\right\|\right)\right]^{p_{k, l}}\right)^{\frac{1}{K}} \\
& \leq\left(\frac{1}{h_{r, s}} \sum_{(k, l) \in I_{r, s}}\left[M_{k, l}\left(\left\|\frac{\Delta^{v} x_{k+m, l+n}}{\rho_{\epsilon}}, z_{1}, \cdots, z_{n-1}\right\|\right)\right]^{p_{k, l}}\right)^{\frac{1}{K}} \leq 1,
\end{aligned}
$$

for each $r, s, m$ and $n$. Suppose that $x_{k, l} \neq 0$ for each $k, l \in N$. This implies that $\Delta^{v} x_{k+m, l+n} \neq 0$, for each $k, l, m, n \in N$. 
Let $\epsilon \rightarrow 0$, then $\left(\left\|\frac{\Delta^{v} x_{k+m, l+n}}{\epsilon}, z_{1}, \cdots, z_{n-1}\right\|\right) \rightarrow \infty$. It follows that

$$
\left(\frac{1}{h_{r, s}} \sum_{(k, l) \in I_{r, s}}\left[M_{k, l}\left(\left\|\frac{\Delta^{v} x_{k+m, l+n}}{\epsilon}, z_{1}, \cdots, z_{n-1}\right\|\right)\right]^{p_{k, l}}\right)^{\frac{1}{K}} \rightarrow \infty,
$$

which is a contradiction. Therefore, $\Delta^{v} x_{k+m, l+n}=0$ for each $k, l, m$ and $n$ and thus $x_{k, l}=0$ for each $k, l \in N$. Let $\rho_{1}>0$ and $\rho_{2}>0$ be such that

$$
\left(\frac{1}{h_{r, s}} \sum_{(k, l) \in I_{r, s}}\left[M_{k, l}\left(\left\|\frac{\Delta^{v} x_{k+m, l+n}}{\rho_{1}}, z_{1}, \cdots, z_{n-1}\right\|\right)\right]^{p_{k, l}}\right)^{\frac{1}{K}} \leq 1
$$

and

$$
\left(\frac{1}{h_{r, s}} \sum_{(k, l) \in I_{r, s}}\left[M_{k, l}\left(\left\|\frac{\Delta^{v} x_{k+m, l+n}}{\rho_{2}}, z_{1}, \cdots, z_{n-1}\right\|\right)\right]^{p_{k, l}}\right)^{\frac{1}{K}} \leq 1
$$

for each $r, s, m$ and $n$. Let $\rho=\rho_{1}+\rho_{2}$. Then, by Minkowski's inequality, we have

$$
\begin{aligned}
( & \left.\frac{1}{h_{r, s}} \sum_{(k, l) \in I_{r, s}}\left[M_{k, l}\left(\left\|\frac{\Delta^{v}\left(x_{k+m, l+n}+y_{k+m, l+n}\right)}{\rho}, z_{1}, \cdots, z_{n-1}\right\|\right)\right]^{p_{k, l}}\right)^{\frac{1}{K}} \\
\leq & \left(\sum _ { ( k , l ) \in I _ { r , s } } \left[\frac{\rho_{1}}{\rho_{1}+\rho_{2}} M_{k, l}\left(\left\|\frac{\Delta^{v}\left(x_{k+m, l+n}\right)}{\rho_{1}}, z_{1}, \cdots, z_{n-1}\right\|\right)\right.\right. \\
& \left.\left.+\frac{\rho_{2}}{\rho_{1}+\rho_{2}} M_{k, l}\left(\left\|\frac{\Delta^{v}\left(y_{k+m, l+n}\right)}{\rho_{2}}, z_{1}, \cdots, z_{n-1}\right\|\right)\right]^{p_{k, l}}\right)^{\frac{1}{K}} \\
\leq & \left(\frac{\rho_{1}}{\rho_{1}+\rho_{2}}\right)\left(\frac{1}{h_{r, s}} \sum_{(k, l) \in I_{r, s}}\left[M_{k, l}\left(\left\|\frac{\Delta^{v}\left(x_{k+m, l+n}\right)}{\rho_{1}}, z_{1}, \cdots, z_{n-1}\right\|\right)\right]^{p_{k, l}}\right)^{\frac{1}{K}} \\
& +\left(\frac{\rho_{2}}{\rho_{1}+\rho_{2}}\right)\left(\frac{1}{h_{r, s}} \sum_{(k, l) \in I_{r, s}}\left[M_{k, l}\left(\left\|\frac{\Delta^{v}\left(y_{k+m, l+n}\right)}{\rho_{2}}, z_{1}, \cdots, z_{n-1}\right\|\right)\right]^{p_{k, l}}\right)^{\frac{1}{K}}
\end{aligned}
$$

$\leq 1$

Since $\rho^{\prime} s$ are non-negative, so we have

$$
\begin{aligned}
& g(x+y)=\inf \left\{\rho^{\frac{p r, s}{K}}:\right. \\
& \begin{aligned}
&\left(\frac{1}{h_{r, s}} \sum_{(k, l) \in I_{r, s}}\right. {\left.\left[M_{k, l}\left(\left\|\frac{\Delta^{v}\left(x_{k+m, l+n}+y_{k+m, l+n}\right)}{\rho}, z_{1}, \cdots, z_{n-1}\right\|\right)\right]^{p_{k, l}}\right)^{\frac{1}{K}} } \\
&\leq 1, r, s \in \mathbb{N}\}, \\
& \leq \inf \left\{\rho_{1}^{\frac{p_{r, s}}{K}}:\left(\frac{1}{h_{r, s}} \sum_{(k, l) \in I_{r, s}}\left[M_{k, l}\left(\left\|\frac{\Delta^{v}\left(x_{k+m, l+n}\right)}{\rho_{1}}, z_{1}, \cdots, z_{n-1}\right\|\right)\right]^{p_{k, l}}\right)^{\frac{1}{K}}\right.
\end{aligned}
\end{aligned}
$$




$$
\begin{aligned}
& \leq 1, r, s \in \mathbb{N}\} \\
& +\inf \left\{\rho_{2}^{\frac{p_{r, s}}{K}}:\left(\frac{1}{h_{r, s}} \sum_{(k, l) \in I_{r, s}}\left[M_{k, l}\left(\left\|\frac{\Delta^{v}\left(y_{k+m, l+n}\right)}{\rho_{2}}, z_{1}, \cdots, z_{n-1}\right\|\right)\right]^{p_{k, l}}\right)^{\frac{1}{K}}\right. \\
& \leq 1, r, s \in \mathbb{N}\} .
\end{aligned}
$$

Therefore,

$$
g(x+y) \leq g(x)+g(y) .
$$

Finally, we prove that the scalar multiplication is continuous. Let $\lambda$ be any complex number. By definition,

$$
\begin{aligned}
& g(\lambda x)=\inf \left\{\rho^{\frac{p_{r, s}}{K}}:\right. \\
& \left.\left(\frac{1}{h_{r, s}} \sum_{(k, l) \in I_{r, s}}\left[M_{k, l}\left(\left\|\frac{\Delta^{v} \lambda x_{k+m, l+n}}{\rho}, z_{1}, \cdots, z_{n-1}\right\|\right)\right]^{p_{k, l}}\right)^{\frac{1}{K}} \leq 1, r, s \in \mathbb{N}\right\} .
\end{aligned}
$$

Then

$$
\begin{aligned}
& g(\lambda x)=\inf \left\{(|\lambda| t)^{\frac{p_{r, s}}{K}}:\right. \\
& \left.\left(\frac{1}{h_{r, s}} \sum_{(k, l) \in I_{r, s}}\left[M_{k, l}\left(\left\|\frac{\Delta^{v} x_{k+m, l+n}}{t}, z_{1}, \cdots, z_{n-1}\right\|\right)\right]^{p_{k, l}}\right)^{\frac{1}{K}} \leq 1, r, s \in \mathbb{N}\right\},
\end{aligned}
$$

where $t=\frac{\rho}{|\lambda|}$. Since $|\lambda|^{p_{r, s}} \leq \max \left(1,|\lambda|^{\text {sup }} p_{r, s}\right)$, we have

$$
\begin{aligned}
& g(\lambda x) \leq \max \left(1,|\lambda|^{\text {sup } p_{r, s}}\right) \inf \left\{t^{\frac{p_{r}}{K}}:\right. \\
& \left.\left(\frac{1}{h_{r, s}} \sum_{(k, l) \in I_{r, s}}\left[M_{k, l}\left(\left\|\frac{\Delta^{v} x_{k+m, l+n}}{t}, z_{1}, \cdots, z_{n-1}\right\|\right)\right]^{p_{k, l}}\right)^{\frac{1}{K}} \leq 1, r, s \in \mathbb{N}\right\} .
\end{aligned}
$$

So, the fact that scalar multiplication is continuous follows from the above inequality. This completes the proof of the theorem.

To prove the next theorem we need the following lemma.

Lemma 1. Let $M$ be an Orlicz function which satisfies $\Delta_{2}$-condition and let $0<$ $\delta<1$. Then for each $x \geq \delta$ we have $M(x)<K \delta^{-1} M(2)$ for some constant $K>0$.

Theorem 3. For a sequence of Orlicz functions $\mathcal{M}=\left(M_{k, l}\right)$ which satisfies $\Delta_{2}$ condition, we have $\left[A C_{\theta_{r, s}}, \Delta^{v},\|\cdot, \cdots, \cdot\|\right] \subseteq\left[A C_{\theta_{r, s}} \mathcal{M}, \Delta^{v},\|\cdot, \cdots, \cdot\|\right]$.

Proof. Let $x=\left(x_{k, l}\right) \in\left[A C_{\theta_{r, s}}, \Delta^{v}\|\cdot, \cdots, \cdot\|\right]$ so that for each $m$ and $n$, we have $D_{r, s}=\left\{x=\left(x_{k, l}\right): P-\lim _{r, s} \frac{1}{h_{r, s}} \sum_{(k, l) \in I_{r, s}}\left\|\frac{\Delta^{v} x_{k+m, l+n}-L}{\rho}, z_{1}, \cdots, z_{n-1}\right\|=0\right.$, 
uniformly in $m$ and $n$ for some $L\}$.

Let $\epsilon>0$ and choose $\delta$ with $0<\delta<1$ such that $M_{k, l}(t)<\epsilon$ for every $t$ with $0 \leq t \leq \delta$.

Now, we have

$$
\begin{aligned}
& \lim _{r, s} \frac{1}{h_{r, s}} \sum_{(k, l) \in I_{r, s}}\left\|\frac{\Delta^{v} x_{k+m, l+n}-L}{\rho}, z_{1}, \cdots, z_{n-1}\right\| \\
& =\lim _{r, s} \frac{1}{h_{r, s}} \sum_{\substack{(k, l) \in I_{r, s} \\
\left\|\frac{\Delta^{v} x_{k+m, l+n}-L}{\rho}, z_{1}, \cdots, z_{n-1}\right\| \leq \delta}} M_{k, l}\left(\left\|\frac{\Delta^{v} x_{k+m, l+n}-L}{\rho}, z_{1}, \cdots, z_{n-1}\right\|\right) \\
& +\lim _{r, s} \frac{1}{h_{r, s}} \sum_{k, l}\left(\left\|\frac{\Delta^{v} x_{k+m, l+n}-L}{\rho}, z_{1}, \cdots, z_{n-1}\right\|\right) \\
& \leq \frac{1}{h_{r, s}}\left(h_{r, s} \epsilon\right)+\lim _{r, s} \frac{1}{h_{r, s}} \sum_{(k, l) \in I_{r, s}}^{\rho}, M_{k, l}\left(\left\|\frac{\Delta^{v} x_{k+m, l+n}-L}{\rho}, z_{1}, \cdots, z_{n-1}\right\|\right) \\
& \left.\sum_{(k, l) \in I_{r, s}} M_{x_{k+m, n}} \|\right) \\
& <\frac{1}{h_{r, s}}\left(h_{r, s} \epsilon\right)+\frac{1}{h_{r, s}} K \delta^{-1} M_{k, l}(2) h_{r, s} D_{r, s} .
\end{aligned}
$$

Therefore by Lemma 1 as $r$ and $s$ goes to infinity in the Pringsheim sense, for each $m$ and $n$, we have $x=\left(x_{k, l}\right) \in\left[A C_{\theta_{r, s}} \mathcal{M}, \Delta^{v}\|\cdot, \cdots, \cdot \mid\|\right]$. This completes the proof of the theorem.

Theorem 4. Let $0<\inf p_{k, l}=h \leq p_{k, l} \leq \sup p_{k, l}=H<\infty$ and $\mathcal{M}=\left(M_{k, l}\right)$, $\mathcal{M}^{\prime}=\left(M_{k, l}^{\prime}\right)$ be two sequences of Orlicz functions which satisfying $\Delta_{2}$-condition, we have
(i) $\left[A C_{\theta_{r, s}}, \mathcal{M}^{\prime}, p, \Delta^{v}\|\cdot, \cdots, \cdot\|\right] \subset\left[A C_{\theta_{r, s}}, \mathcal{M} \circ \mathcal{M}^{\prime}, p, \Delta^{v}\|\cdot, \cdots, \cdot\|\right]$ and
(ii) $\left[A C_{\theta_{r, s}}, \mathcal{M}^{\prime}, p, \Delta^{v}\|\cdot, \cdots, \cdot\|\right]_{0} \subset\left[A C_{\theta_{r, s}}, \mathcal{M} \circ \mathcal{M}^{\prime}, p, \Delta^{v}\|\cdot, \cdots, \cdot\|\right]_{0}$.

Proof. Let $x=\left(x_{k, l}\right) \in\left[A C_{\theta_{r, s},}, \mathcal{M}^{\prime}, p, \Delta^{v}\|\cdot, \cdots, \cdot\|\right]$. Then we have

$$
\lim _{r, s} \frac{1}{h_{r, s}} \sum_{(k, l) \in I_{r, s}}\left[M_{k, l}^{\prime}\left(\left\|\frac{\Delta^{v} x_{k+m, l+n}-L}{\rho}, z_{1}, \cdots, z_{n-1}\right\|\right)\right]^{p_{k, l}}=0
$$

uniformly in $m$ and $n$ for some $L$ and $\rho>0$. 
Let $\epsilon>0$ and choose $\delta$ with $0<\delta<1$ such that $M_{k, l}(t)<\epsilon$ for $0 \leq t \leq \delta$. Let

$$
y_{k, l}=M_{k, l}^{\prime}\left(\left\|\frac{\Delta^{v} x_{k+m, l+n}-L}{\rho}, z_{1}, \cdots, z_{n-1}\right\|\right) \text { for all } k, l \in \mathbb{N} .
$$

We can write

$$
\begin{aligned}
\frac{1}{h_{r, s}} \sum_{(k, l) \in I_{r, s}}\left[M_{k, l}\left(y_{k, l}\right)\right]^{p_{k, l}}= & \frac{1}{h_{r, s}} \sum_{(k, l) \in I_{r, s}, y_{k, l} \leq \delta}\left[M_{k, l}\left(y_{k, l}\right)\right]^{p_{k, l}} \\
& +\frac{1}{h_{r, s}} \sum_{(k, l) \in I_{r, s}, y_{k, l>\delta}}\left[M_{k, l}\left(y_{k, l}\right)\right]^{p_{k, l} .} .
\end{aligned}
$$

Since $\mathcal{M}=\left(M_{k, l}\right)$ satisfies $\Delta_{2}$-condition, we have

$$
\begin{aligned}
& \frac{1}{h_{r, s}} \sum_{(k, l) \in I_{r, s}, y_{k, l} \leq \delta} {\left[M_{k, l}\left(y_{k, l}\right)\right]^{p_{k, l}} } \\
& \leq\left[M_{k, l}(1)\right]^{H} \frac{1}{h_{r, s}} \sum_{(k, l) \in I_{r, s}, y_{k, l} \leq \delta}\left[M_{k, l}\left(y_{k, l}\right)\right]^{p_{k, l}} \\
& \leq\left[M_{k, l}(2)\right]^{H} \frac{1}{h_{r, s}} \sum_{(k, l) \in I_{r, s}, y_{k, l} \leq \delta}\left[M_{k, l}\left(y_{k, l}\right)\right]^{p_{k, l}}
\end{aligned}
$$

For $y_{k, l}>\delta$

$$
y_{k, l}<\frac{y_{k, l}}{\delta}<1+\frac{y_{k, l}}{\delta} .
$$

Since $\mathcal{M}=\left(M_{k, l}\right)$ is non-decreasing and convex, it follows that

$$
M_{k, l}\left(y_{k, l}\right)<M_{k, l}\left(1+\frac{y_{k, l}}{\delta}\right)<\frac{1}{2} M_{k, l}(2)+\frac{1}{2} M_{k, l}\left(\frac{2 y_{k, l}}{\delta}\right) .
$$

Also $\left(M_{k, l}\right)$ satisfies $\Delta_{2}$-condition, we can write

$$
M_{k, l}\left(y_{k, l}\right)<\frac{1}{2} T \frac{y_{k, l}}{\delta} M_{k, l}(2)+\frac{1}{2} T \frac{y_{k, l}}{\delta} M_{k, l}(2)=T \frac{y_{k, l}}{\delta} M_{k, l}(2) .
$$

Hence,

$$
\begin{aligned}
\frac{1}{h_{r, s}} \sum_{(k, l) \in I_{r, s}, y_{k, l>\delta}} & {\left[M_{k, l}\left(y_{k, l}\right)\right]^{p_{k, l}} } \\
& \leq \max \left(1,\left(\frac{T M_{k, l}(2)}{\delta}\right)^{H}\right) \frac{1}{h_{r, s}} \sum_{(k, l) \in I_{r, s}, y_{k, l}>\delta}\left[\left(y_{k, l}\right)\right]^{p_{k, l}}
\end{aligned}
$$

From equations (2.1) and (2.2), we have

$$
x=\left(x_{k, l}\right) \in\left[A C_{\theta_{r, s}}, \mathcal{M} \circ \mathcal{M}^{\prime}, p, \Delta^{v}\|\cdot, \cdots, \cdot\|\right] .
$$


This completes the proof of (i). Similarly, we can prove that $\left[A C_{\theta_{r, s}}, \mathcal{M}^{\prime}, p, \Delta^{v}\|\cdot, \cdots, \cdot\|\right]_{0} \subset\left[A C_{\theta_{r, s}}, \mathcal{M} \circ \mathcal{M}^{\prime}, p, \Delta^{v}\|\cdot, \cdots, \cdot\|\right]_{0}$.

Theorem 5. If $0<p_{k, l} \leq q_{k, l}<\infty$ for all $k, l \in \mathbb{N}$ and $\left(\frac{q_{k, l}}{p_{k, l}}\right)$ be bounded, then $\left[A C_{\theta_{r, s}}, \mathcal{M} \circ \mathcal{M}^{\prime}, q, \Delta^{v}\|\cdot, \cdots, \cdot\|\right] \subset\left[A C_{\theta_{r, s}}, \mathcal{M} \circ \mathcal{M}^{\prime}, p, \Delta^{v}\|\cdot, \cdots, \cdot\|\right]$.

Proof. Let $x=\left(x_{k, l}\right) \in\left[A C_{\theta_{r, s}}, \mathcal{M} \circ \mathcal{M}^{\prime}, q, \Delta^{v}\|\cdot, \cdots, \cdot\|\right]$. Write

$$
t_{k, l}=\left[M_{k, l}\left(\left\|\frac{\Delta^{v} x_{k+m, l+n}-L}{\rho}, z_{1}, \cdots, z_{n-1}\right\|\right)\right]^{q_{k, l}}
$$

and $\mu_{k, l}=\frac{p_{k, l}}{q_{k, l}}$ for all $k, l \in \mathbb{N}$. Then $0<\mu_{k, l} \leq 1$ for $k, l \in \mathbb{N}$. Take $0<\mu<\mu_{k, l}$ for $k, l \in \mathbb{N}$. Define the sequences $\left(u_{k, l}\right)$ and $\left(v_{k, l}\right)$ as follows: For $t_{k, l} \geq 1$, let $u_{k, l}=t_{k, l}$ and $v_{k, l}=0$ and for $t_{k, l}<1$, let $u_{k, l}=0$ and $v_{k, l}=t_{k, l}$. Then clearly for all $k, l \in \mathbb{N}$, we have

$$
t_{k, l}=u_{k, l}+v_{k, l}, \quad t_{k, l}^{\mu_{k, l}}=u_{k, l}^{\mu_{k, l}}+v_{k, l}^{\mu_{k, l}}
$$

Now it follows that $u_{k, l}^{\mu_{k, l}} \leq u_{k, l} \leq t_{k, l}$ and $v_{k, l}^{\mu_{k, l}} \leq v_{k, l}^{\mu}$. Therefore,

$$
\begin{aligned}
\frac{1}{h_{r, s}} \sum_{(k, l) \in I_{r, s}} t_{k, l}^{\mu_{k, l}} & =\frac{1}{h_{r, s}} \sum_{(k, l) \in I_{r, s}}\left(u_{k, l}^{\mu_{k, l}}+v_{k, l}^{\mu_{k, l}}\right) \\
& \leq \frac{1}{h_{r, s}} \sum_{(k, l) \in I_{r, s}} t_{k, l}+\frac{1}{h_{r, s}} \sum_{(k, l) \in I_{r, s}} v_{k, l}^{\mu} .
\end{aligned}
$$

Now for each $k, l$ we have

$$
\begin{aligned}
\frac{1}{h_{r, s}} \sum_{(k, l) \in I_{r, s}} v_{k, l}^{\mu} & =\sum_{(k, l) \in I_{r, s}}\left(\frac{1}{h_{r, s}} v_{k, l}\right)^{\mu}\left(\frac{1}{h_{r, s}}\right)^{1-\mu} \\
& \leq\left(\sum_{(k, l) \in I_{r, s}}\left[\left(\frac{1}{h_{r, s}} v_{k, l}\right)^{\mu}\right]^{\frac{1}{\mu}}\right)^{\mu}\left(\sum_{(k, l) \in I_{r, s}}\left[\left(\frac{1}{h_{r, s}}\right)^{1-\mu}\right]^{\frac{1}{1-\mu}}\right)^{1-\mu} \\
& =\left(\frac{1}{h_{r, s}} \sum_{(k, l) \in I_{r, s}} v_{k, l}\right)^{\mu}
\end{aligned}
$$

and so

$$
\frac{1}{h_{r, s}} \sum_{(k, l) \in I_{r, s}} t_{k, l}^{\mu_{k, l}} \leq \frac{1}{h_{r, s}} \sum_{(k, l) \in I_{r, s}} t_{k, l}+\left(\frac{1}{h_{r, s}} \sum_{(k, l) \in I_{r, s}} v_{k, l}\right)^{\mu} .
$$

Hence $x=\left(x_{k, l}\right) \in\left[A C_{\theta_{r, s}}, \mathcal{M} \circ \mathcal{M}^{\prime}, p, \Delta^{v}\|\cdot, \cdots, \cdot\|\right]$. 


\section{INCLUSION RELATIONS}

Let $\ell_{\infty}, c$ and $c_{0}$ denotes the sequence spaces of bounded, convergent and null sequences $x=\left(x_{k}\right)$ respectively. A sequence $x=\left(x_{k}\right) \in \ell_{\infty}$ is said to be almost convergent if all Banach limits of $x=\left(x_{k}\right)$ coincide. In [19], it was shown that

$$
f=\left\{x=\left(x_{k}\right): \lim _{n \rightarrow \infty} \frac{1}{n} \sum_{k=1}^{n} x_{k+s} \text { exists, uniformly in } s\right\} .
$$

In [19] Maddox defined strongly almost convergent sequences. Recall that a sequence $x=\left(x_{k}\right)$ is strongly almost convergent if there is a number $L$ such that

$$
\lim _{n \rightarrow \infty} \frac{1}{n} \sum_{k=1}^{n}\left|x_{k+s}-L\right|=0, \text { uniformly in } s .
$$

Let $\mathcal{M}=\left(M_{k, l}\right)$ be a sequence of Orlicz functions and $p=\left(p_{k, l}\right)$ be any factorable double sequence of strictly positive real numbers. Then we define the following sequence spaces:

$$
\begin{aligned}
& {\left[\mathcal{F}, \mathcal{M}, p, \Delta^{v},\|\cdot, \cdots, \cdot\|\right]=\left\{x=\left(x_{k, l}\right) \in w(X):\right.} \\
& \quad P-\lim _{p, q} \frac{1}{p q} \sum_{k, l=1,1}^{p, q}\left[M_{k, l}\left(\left\|\frac{\Delta^{v} x_{k+m, l+n}-L}{\rho}, z_{1}, \cdots, z_{n-1}\right\|\right)\right]^{p_{k, l}}=0, \\
& \quad \text { uniformly in } m \text { and } n \text {, for some } L \text { and } \rho>0\}
\end{aligned}
$$

and

$$
\begin{aligned}
& {\left[\mathcal{F}, \mathcal{M}, p, \Delta^{v},\|\cdot, \cdots, \cdot\|\right]_{0}=\left\{x=\left(x_{k, l}\right) \in w(X):\right.} \\
& \quad P-\lim _{p, q} \frac{1}{p q} \sum_{k, l=1,1}^{p, q}\left[M_{k, l}\left(\left\|\frac{\Delta^{v} x_{k+m, l+n}}{\rho}, z_{1}, \cdots, z_{n-1}\right\|\right)\right]^{p_{k, l}}=0, \\
& \text { uniformly in } m \text { and } n \text {, for some } \rho>0\} .
\end{aligned}
$$

If we take $\mathcal{M}(x)=x$, we have

$$
\begin{aligned}
& {\left[\mathcal{F}, p, \Delta^{v},\|\cdot, \cdots, \cdot\|\right]=\left\{x=\left(x_{k, l}\right) \in w(X):\right.} \\
& \quad P-\lim _{p, q} \frac{1}{p q} \sum_{k, l=1,1}^{p, q}\left(\left\|\frac{\Delta^{v} x_{k+m, l+n}-L}{\rho}, z_{1}, \cdots, z_{n-1}\right\|\right)^{p_{k, l}}=0, \\
& \quad \text { uniformly in } m \text { and } n, \text { for some } L \text { and } \rho>0\}
\end{aligned}
$$

and

$$
\left[\mathcal{F}, p, \Delta^{v},\|\cdot, \cdots, \cdot\|\right]_{0}=\left\{x=\left(x_{k, l}\right) \in w(X):\right.
$$




$$
P-\lim _{p, q} \frac{1}{p q} \sum_{k, l=1,1}^{p, q}\left(\left\|\frac{\Delta^{v} x_{k+m, l+n}}{\rho}, z_{1}, \cdots, z_{n-1}\right\|\right)^{p_{k, l}}=0,
$$

uniformly in $m$ and $n$, for some $\rho>0\}$.

If we take $p=\left(p_{k, l}\right)=1$, we get

$$
\begin{aligned}
& {\left[\mathcal{F}, \mathcal{M}, \Delta^{v},\|\cdot, \cdots, \cdot\|\right] }=\left\{x=\left(x_{k, l}\right) \in w(X):\right. \\
& P-\lim _{p, q} \frac{1}{p q} \sum_{k, l=1,1}^{p, q} M_{k, l}\left(\left\|\frac{\Delta^{v} x_{k+m, l+n}-L}{\rho}, z_{1}, \cdots, z_{n-1}\right\|\right)=0,
\end{aligned}
$$

uniformly in $m$ and $n$, for some $L$ and $\rho>0\}$

and

$$
\begin{aligned}
& {\left[\mathcal{F}, \mathcal{M}, \Delta^{v},\|\cdot, \cdots, \cdot\|\right]_{0}=\left\{x=\left(x_{k, l}\right) \in w(X):\right.} \\
& P-\lim _{p, q} \frac{1}{p q} \sum_{k, l=1,1}^{p, q} M_{k, l}\left(\left\|\frac{\Delta^{v} x_{k+m, l+n}}{\rho}, z_{1}, \cdots, z_{n-1}\right\|\right)=0, \\
& \text { uniformly in } m \text { and } n, \text { for some } \rho>0\} .
\end{aligned}
$$

If we take $\mathcal{M}(x)=x$ and $p=\left(p_{k, l}\right)=1$, we get

$$
\begin{aligned}
& {\left[\mathcal{F}, \Delta^{v},\|\cdot, \cdots, \cdot\|\right]=\left\{x=\left(x_{k, l}\right) \in w(X):\right.} \\
& \quad P-\lim _{p, q} \frac{1}{p q} \sum_{k, l=1,1}^{p, q}\left(\left\|\frac{\Delta^{v} x_{k+m, l+n}-L}{\rho}, z_{1}, \cdots, z_{n-1}\right\|\right)=0, \\
& \text { uniformly in } m \text { and } n, \text { for some } L \text { and } \rho>0\}
\end{aligned}
$$

and

$$
\begin{aligned}
& {\left[\mathcal{F}, \Delta^{v},\|\cdot, \cdots, \cdot\|\right]_{0}=\left\{x=\left(x_{k, l}\right) \in w(X):\right.} \\
& \quad P-\lim _{p, q} \frac{1}{p q} \sum_{k, l=1,1}^{p, q}\left(\left\|\frac{\Delta^{v} x_{k+m, l+n}}{\rho}, z_{1}, \cdots, z_{n-1}\right\|\right)=0, \\
& \quad \text { uniformly in } m \text { and } n, \text { for some } \rho>0\} .
\end{aligned}
$$

In this section of the paper we study inclusion relations between the spaces $\left[A C_{\theta_{r, s}}, \mathcal{M}, p, \Delta^{v},\|\cdot, \cdots, \cdot\|\right]_{0},\left[\mathcal{F}, \mathcal{M}, p, \Delta^{v},\|\cdot, \cdots, \cdot\|\right]$ and $\left[\mathcal{F}, \mathcal{M}, p, \Delta^{v},\|\cdot, \cdots, \cdot\|\right]$. 
Theorem 6. Let $\mathcal{M}=\left(M_{k, l}\right)$ be a sequence of Orlicz functions and $p=\left(p_{k, l}\right)$ be a factorable double sequence of strictly positive real numbers, then the spaces $\left[\widetilde{F}, \mathcal{M}, \Delta^{v}, p,\|\cdot, \cdots, \cdot\|\right]$ and $\left[\mathcal{F}, \mathcal{M}, \Delta^{v}, p,\|\cdot, \cdots, \cdot\|\right]_{0}$ are linear spaces over the field of complex number $\mathbb{C}$.

Proof. It is easy to prove.

Theorem 7. For any sequence of Orlicz functions $\mathcal{M}=\left(M_{k, l}\right)$ and $p=\left(p_{k, l}\right)$ be a factorable double sequence of positive real numbers, the space $\left[\mathcal{F}, \mathcal{M}, \Delta^{v}, p,\|\cdot, \cdots, \cdot\|\right]$ is a topological linear space paranormed by $g(x)=\inf \left\{\rho^{\frac{p p, q}{K}}:\left(\frac{1}{p q} \sum_{k, l=1,1}^{p, q}\left[M_{k, l}\left(\left\|\frac{\Delta^{v} x_{k+m, l+n}}{\rho}, z_{1}, \cdots, z_{n-1}\right\|\right)\right]^{p_{k, l}}\right)^{\frac{1}{K}} \leq 1\right\}$, where $K=\max \left(1, \sup _{k, l} p_{k, l}<\infty\right)$.

Proof. It is easy to prove in view of Theorem 2, so we omit the details.

Theorem 8. Let $\mathcal{M}=\left(M_{k, l}\right)$ be a sequence of Orlicz functions and $\theta_{r, s}=\left\{k_{r}, l_{s}\right\}$ be a double lacunary sequence with $\liminf _{r} q_{r}>1$ and $\liminf _{s} \bar{q}_{s}>1$, we have (i) $\left[\mathcal{F}, \mathcal{M}, \Delta^{v}, p,\|\cdot, \cdots, \cdot\|\right]_{0} \subset\left[A C_{\theta_{r, s}}, \mathcal{M}, \Delta^{v}, p,\|\cdot, \cdots, \cdot\|\right]_{0}$, (ii) $\left[\mathcal{F}, \mathcal{M}, \Delta^{v}, p,\|\cdot, \cdots, \cdot\|\right] \subset\left[A C_{\theta_{r, s}}, \mathcal{M}, \Delta^{v}, p,\|\cdot, \cdots, \cdot\|\right]$.

Proof. Let $\liminf _{r} q_{r}>1$ and $\liminf _{s} \bar{q}_{s}>1$, then there exists $\delta>0$ such that $q_{r}>$ $1+\delta$ and $\bar{q}_{s}>1+\delta$. This implies $\frac{h_{r}}{k_{r}} \geq \frac{\delta}{1+\delta}$ and $\frac{\bar{h}_{s}}{l_{s}} \geq \frac{\delta}{1+\delta}$. Then for $x=\left(x_{k, l}\right) \in$ $\left[\mathcal{F}, \mathcal{M}, \Delta^{v}, p,\|\cdot, \cdots, \cdot\|\right]_{0}$, we can write for each $m$ and $n$

$$
\begin{aligned}
B_{r, s}= & \frac{1}{h_{r, s}} \sum_{(k, l) \in I_{r, s}}\left[M_{k, l}\left(\left\|\frac{\Delta^{v} x_{k+m, l+n}}{\rho}, z_{1}, \cdots, z_{n-1}\right\|\right)\right]^{p_{k, l}} \\
= & \frac{1}{h_{r, s}} \sum_{k=1}^{k_{r}} \sum_{l=1}^{l_{s}}\left[M_{k, l}\left(\left\|\frac{\Delta^{v} x_{k+m, l+n}}{\rho}, z_{1}, \cdots, z_{n-1}\right\|\right)\right]^{p_{k, l}} \\
& -\frac{1}{h_{r, s}} \sum_{k=1}^{k_{r-1}} \sum_{l=1}^{l_{s-1}}\left[M_{k, l}\left(\left\|\frac{\Delta^{v} x_{k+m, l+n}}{\rho}, z_{1}, \cdots, z_{n-1}\right\|\right)\right]^{p_{k, l}} \\
& -\frac{1}{h_{r, s}} \sum_{k=k_{r-1}+1}^{k_{r}} \sum_{l=1}^{l_{s-1}}\left[M_{k, l}\left(\left\|\frac{\Delta^{v} x_{k+m, l+n}}{\rho}, z_{1}, \cdots, z_{n-1}\right\|\right)\right]^{p_{k, l}} \\
& -\frac{1}{h_{r, s}} \sum_{k=1}^{k_{r-1}} \sum_{l=l_{s-1}+1}^{l_{s}}\left[M_{k, l}\left(\left\|\frac{\Delta^{v} x_{k+m, l+n}}{\rho}, z_{1}, \cdots, z_{n-1}\right\|\right)\right]^{p_{k, l}}
\end{aligned}
$$




$$
\begin{aligned}
= & \frac{k_{r} k_{s}}{h_{r s}}\left(\frac{1}{k_{r} l_{s}} \sum_{k=1}^{k_{r}} \sum_{l=1}^{l_{s}}\left[M_{k, l}\left(\left\|\frac{\Delta^{v} x_{k+m, l+n}}{\rho}, z_{1}, \cdots, z_{n-1}\right\|\right)\right]^{p_{k, l}}\right) \\
& -\frac{k_{r-1} l_{s-1}}{h_{r, s}}\left(\frac{1}{k_{r-1} l_{s-1}} \sum_{k=1}^{k_{r-1}} \sum_{l=1}^{l_{s-1}}\left[M_{k, l}\left(\left\|\frac{\Delta^{v} x_{k+m, l+n}}{\rho}, z_{1}, \cdots, z_{n-1}\right\|\right)\right]^{p_{k, l}}\right) \\
& -\frac{1}{h_{r}} \sum_{k=k_{r-1}+1}^{k_{r}} \frac{l_{s-1}}{h_{s}} \frac{1}{l_{s-1}} \sum_{l=1}^{l_{s-1}}\left[M_{k, l}\left(\left\|\frac{\Delta^{v} x_{k+m, l+n}}{\rho}, z_{1}, \cdots, z_{n-1}\right\|\right)\right]^{p_{k, l}} \\
& -\frac{1}{h_{s}} \sum_{l=l_{s-1}+1}^{l_{s}} \frac{k_{r-1}}{h_{r}} \frac{1}{k_{r-1}} \sum_{k=1}^{k_{r-1}}\left[M_{k, l}\left(\left\|\frac{\Delta^{v} x_{k+m, l+n}}{\rho}, z_{1}, \cdots, z_{n-1}\right\|\right)\right]^{p_{k, l}} .
\end{aligned}
$$

Since $x=\left(x_{k, l}\right) \in\left[\mathscr{F}, \mathcal{M}, \Delta^{v}, p,\|\cdot, \cdots, \cdot\|\right]$ the last two terms tends to zero uniformly in $m, n$ in the Pringsheim sense, thus for each $m$ and $n$, we have

$$
\begin{aligned}
& B_{r, s}=\frac{k_{r} k_{s}}{h_{r s}}\left(\frac{1}{k_{r} l_{s}} \sum_{k=1}^{k_{r}} \sum_{l=1}^{k_{s}}\left[M_{k, l}\left(\left\|\frac{\Delta^{v} x_{k+m, l+n}}{\rho}, z_{1}, \cdots, z_{n-1}\right\|\right)\right]^{p_{k, l}}\right) \\
& -\frac{k_{r-1} l_{s-1}}{h_{r s}}\left(\frac{1}{k_{r-1} l_{s-1}} \sum_{k=1}^{k_{r-1}} \sum_{l=1}^{l_{s-1}}\left[M_{k, l}\left(\left\|\frac{\Delta^{v} x_{k+m, l+n}}{\rho}, z_{1}, \cdots, z_{n-1}\right\|\right)\right]^{p_{k, l}}\right)+o(1) .
\end{aligned}
$$

Since $h_{r s}=k_{r} l_{s}-k_{r-1} l_{s-1}$ we are granted for each $m$ and $n$ the following:

$$
\frac{k_{r} l_{s}}{h_{r s}} \leq \frac{1+\delta}{\delta} \text { and } \frac{k_{r-1} l_{s-1}}{h_{r s}} \leq \frac{1}{\delta}
$$

The terms

$$
\frac{1}{k_{r} l_{s}} \sum_{k=1}^{k_{r}} \sum_{l=1}^{l_{s}}\left[M_{k, l}\left(\left\|\frac{\Delta^{v} x_{k+m, l+n}}{\rho}, z_{1}, \cdots, z_{n-1}\right\|\right)\right]^{p_{k, l}}
$$

and

$$
\frac{1}{k_{r-1} l_{s-1}} \sum_{k=1}^{k_{r-1}} \sum_{l=1}^{l_{s-1}}\left[M_{k, l}\left(\left\|\frac{\Delta^{v} x_{k+m, l+n}}{\rho}, z_{1}, \cdots, z_{n-1}\right\|\right)\right]^{p_{k, l}}
$$

are both Pringsheim null sequences for all $m$ and $n$. Thus $B_{r, s}$ is a Pringsheim null sequence for each $m$ and $n$. Therefore $x=\left(x_{k, l}\right) \in\left[A C_{\theta_{r, s}}, \mathcal{M}, \Delta^{v}, p,\|\cdot, \cdots, \cdot\|\right]_{0}$. This completes the proof of (i). Similarly, we can prove (ii). 
Theorem 9. Let $\mathcal{M}=\left(M_{k, l}\right)$ be a sequence of Orlicz functions and $\theta_{r, s}=\left\{k_{r}, l_{s}\right\}$ be a double lacunary sequence with $\lim \sup q_{r}<\infty$ and $\lim \sup \bar{q}_{s}<\infty$, we have

(i) $\left[A C_{\theta_{r, s}}, \mathcal{M}, \Delta^{v}, p,\|\cdot, \cdots, \cdot\|\right]_{0} \subset\left[\mathcal{F}^{r}, \mathcal{M}, \Delta^{v}, p,\|\cdot, \cdots, \cdot\|\right]_{0}^{s}$,

(ii) $\left[A C_{\theta_{r, s}}, \mathcal{M}, \Delta^{v}, p,\|\cdot, \cdots, \cdot\|\right] \subset\left[\mathcal{F}, \mathcal{M}, \Delta^{v}, p,\|\cdot, \cdots, \cdot\|\right]$.

Proof. Since $\limsup { }_{r} q_{r}<\infty$ and $\limsup _{s} \bar{q}_{s}<\infty$ there exists $H>0$ such that $q_{r}<H$ and $\bar{q}_{s}<H$ for all $r$ and $s$. Let $x=\left(x_{k, l}\right) \in\left[A C_{\theta_{r, s}}, \mathcal{M}, \Delta^{v}, p,\|\cdot, \cdots, \cdot\|\right]_{0}$ and $\epsilon>0$. Also there exist $r_{0}>0$ and $s_{0}>0$ such that for every $i \geq r_{0}$ and $j \geq s_{0}$ and all $m$ and $n$, we have

$$
A_{i, j}^{\prime}=\frac{1}{h_{i j}} \sum_{(k, l) \in I_{i, j}}\left[M_{k, l}\left(\left\|\frac{\Delta^{v} x_{k+m, l+n}}{\rho}, z_{1}, \cdots, z_{n-1}\right\|\right)\right]^{p_{k, l}}<\epsilon .
$$

Let $N=\max \left\{A_{i j}^{\prime}: 1 \leq i \leq r_{0}\right.$ and $\left.1 \leq j \leq s_{0}\right\}, p$ and $q$ be such that $k_{r-1}<p \leq k_{r}$ and $l_{s-1}<q \leq l_{s}$. Thus we obtain the following:

$$
\begin{aligned}
& \frac{1}{p q} \sum_{k, l=1,1}^{p, q}\left[M_{k, l}\left(\left\|\frac{\Delta^{v} x_{k_{m}, l+n}}{\rho}, z_{1}, \cdots, z_{n-1}\right\|\right)\right]^{p_{k, l}} \\
& \leq \frac{1}{k_{r-1} l_{s-1}} \sum_{k, l=1,1}^{k_{r} l_{s}}\left[M_{k, l}\left(\left\|\frac{\Delta^{v} x_{k+m, l+n}}{\rho}, z_{1}, \cdots, z_{n-1}\right\|\right)\right]^{p_{k, l}} \\
& \leq \frac{1}{k_{r-1} l_{s-1}} \sum_{t, u=1,1}^{r, s}\left(\sum_{k, l \in I_{t, u}}\left[M_{k, l}\left(\left\|\frac{\Delta^{v} x_{k+m, l+n}}{\rho}, z_{1}, \cdots, z_{n-1}\right\|\right)\right]^{p_{k, l}}\right) \\
& =\frac{1}{k_{r-1} l_{s-1}} \sum_{t, u 1,1}^{r_{0}, s_{0}} h_{t, u} A_{t, u}^{\prime}+\frac{1}{k_{r-1} l_{s-1}} \sum_{\left(r_{0}<t \leq r\right) \cup\left(s_{0}<u \leq s\right)} h_{t, u} A_{t, u}^{\prime} \\
& \leq \frac{N}{k_{r-1} l_{s-1}} \sum_{t, u=1,1}^{r_{0}, s_{0}} h_{t, u}+\frac{1}{k_{r-1} l_{s-1}} \sum_{\left(r_{0}<t \leq r\right) \cup\left(s_{0}<u \leq s\right)} h_{t, u} A_{t, u}^{\prime} \\
& \leq \frac{N k_{r_{0}} l_{s_{0}} r_{0} s_{0}}{k_{r-1} l_{s-1}}+\frac{1}{k_{r-1} l_{s-1}} \sum_{\left(r_{0}<t \leq r\right) \cup\left(s_{0}<u \leq s\right)} h_{t, u} A_{t, u}^{\prime} \\
& \leq \frac{N k_{r_{0}} l_{s_{0}} r_{0} s_{0}}{k_{r-1} l_{s-1}}+\left(\sup _{t \geq r_{0} \cup u \geq s_{0}} A_{t, u}^{\prime} \frac{1}{k_{r-1} l_{s-1}} \sum_{\left(r_{0}<t \leq r\right) \cup\left(s_{0}<u \leq s\right)} h_{t, u}\right. \\
& \leq \frac{N k_{r_{0}} l_{s_{0}} r_{0} s_{0}}{k_{r-1} l_{s-1}}+\frac{1}{k_{r-1} l_{s-1}} \epsilon \sum_{\left(r_{0}<t \leq r\right) \cup\left(s_{0}<u \leq s\right)} h_{t, u}
\end{aligned}
$$




$$
\leq \frac{N k_{r_{0}} l_{s_{0}} r_{0} s_{0}}{k_{r-1} l_{s-1}}+\epsilon H^{2} .
$$

Since $k_{r}$ and $l_{s}$ both approaches infinity as both $p$ and $q$ approaches infinity, it follows that

$$
\frac{1}{p q} \sum_{k, l=1,1}^{p, q}\left[M_{k, l}\left(\left\|\frac{\Delta^{v} x_{k+m, l+n}}{\rho}, z_{1}, \cdots, z_{n-1}\right\|\right)\right]^{p_{k, l}} \rightarrow 0 \text {, uniformly in } m \text { and } n \text {. }
$$

Therefore $x=\left(x_{k, l}\right) \in\left[\mathcal{F}, \mathcal{M}, \Delta^{v}, p,\|\cdot, \cdots, \cdot\|\right]$. This completes the proof of (i). Similarly, we can prove (ii).

Theorem 10. Let $\mathcal{M}=\left(M_{k, l}\right)$ be a sequence of Orlicz functions and $\theta_{r, s}=\left\{k_{r}, l_{s}\right\}$ be a double lacunary sequence with $1<\liminf q_{r, s} \leq \lim \sup _{r, s} q_{r, s}<\infty$, we have

(i) $\left[A C_{\theta_{r, s}}, \mathcal{M}, \Delta^{v}, p,\|\cdot, \cdots, \cdot\|\right]_{0}=\left[\mathcal{F}, \mathcal{M}, \Delta^{v}, p,\|\cdot, \cdots, \cdot\|\right]_{0}$
(ii) $\left[A C_{\theta_{r, s}}, \mathcal{M}, \Delta^{v}, p,\|\cdot, \cdots, \cdot\|\right]=\left[\mathcal{F}, \mathcal{M}, \Delta^{v}, p,\|\cdot, \cdots, \cdot\|\right]$.

Proof. The proof directly follows from Theorem 8 and Theorem 9.

\section{Statistical CONVERGENCE}

The notion of statistical convergence was introduced by Fast [8] and Schoenberg [34] independently. Over the years and under different names, statistical convergence has been discussed in the theory of Fourier analysis, ergodic theory and number theory. Later on, it was further investigated from the sequence space point of view and linked with summability theory by Fridy [9], Connor [6], Salat [32], Mursaleen et al [25], Mursaleen and Edely [27], Mursaleen and Mohiuddine [31], Isık [16], Savas [33], Kolk [18], Maddox [19], Mursaleen et al [26] and many others. In recent years, generalizations of statistical convergence have appeared in the study of strong integral summability and the structure of ideals of bounded continuous functions on locally compact spaces. Statistical convergence and its generalizations are also connected with subsets of the Stone-Cech compactification of natural numbers. Moreover, statistical convergence is closely related to the concept of convergence in probability. The notion depends on the density of subsets of the set $\mathbb{N}$ of natural numbers. A subset $E$ of $\mathbb{N}$ is said to have the natural density $\delta(E)$ if the following limit exists: $\delta(E)=\lim _{n \rightarrow \infty} \frac{1}{n} \sum_{k=1}^{n} \chi_{E}(k)$, where $\chi_{E}$ is the characteristic function of $E$. It is clear that any finite subset of $\mathbb{N}$ has zero natural density and $\delta\left(E^{c}\right)=1-\delta(E)$.

A sequence $x=\left(x_{k, l}\right)$ is said to be lacunary $\Delta^{v}$-statistically convergent to $L$, if for every $\epsilon>0$

$$
\lim _{r, s} \frac{1}{h_{r, s}}\left|\left\{(k, l) \in I_{r, s}:\left\|\left(\Delta^{v} x_{k, l}-L\right), z_{1}, \cdots, z_{n-1}\right\| \geq \epsilon\right\}\right|=0 .
$$


In this case we write $x_{k, l} \rightarrow L\left(S_{\theta_{r, s}}\left(\Delta^{v}\right)\right)$. The set of all lacunary $\Delta^{v}$-statistically convergent sequences is denoted by $S_{\theta_{r, s}}\left(\Delta^{v}\right)$.

Theorem 11. Let $\mathcal{M}=\left(M_{k, l}\right)$ be a sequence of Orlicz functions and $0<h=$ $\inf _{k, l} p_{k, l} \leq p_{k, l} \leq \sup _{k, l} p_{k, l}=H<\infty$. Then $\left[A C_{\theta_{r, s}}, \mathcal{M}, \Delta^{v}, p,\|\cdot, \cdots, \cdot\|\right]$ $\subset S_{\theta_{r, s}}\left(\Delta^{v}\right)$.

Proof. Let $x=\left(x_{k, l}\right) \in\left[A C_{\theta_{r, s}}, \mathcal{M}, \Delta^{v}, p,\|\cdot, \cdots, \cdot\|\right]$ and $\epsilon>0$ be given. Then

$$
\begin{aligned}
& \frac{1}{h_{r, s}} \sum_{(k, l) \in I_{r, s}}\left[M_{k, l}\left(\left\|\frac{\Delta^{v} x_{k, l}-L}{\rho}, z_{1}, \cdots, z_{n-1}\right\|\right)\right]^{p_{k, l}} \\
& \geq \frac{1}{h_{r, s}} \sum_{(k, l) \in I_{r, s},\left(\left\|\frac{\Delta^{v} x_{k, l}-L}{\rho}, z_{1}, \cdots, z_{n-1}\right\|\right) \geq \epsilon}\left[M_{k, l}\left(\left\|\frac{\left(\Delta^{v} x_{k, l}-L\right)}{\rho}, z_{1}, \cdots, z_{n-1}\right\|\right)\right]^{p_{k, l}} \\
& \geq \frac{1}{h_{r, s}} \sum_{(k, l) \in I_{r, s},\left(\left\|\frac{\Delta^{v} x_{k, l}-L}{\rho}, z_{1}, \cdots, z_{n-1}\right\|\right) \geq \epsilon}\left[M_{k, l}(\epsilon)\right]^{p_{k, l}} \\
& \geq \frac{1}{h_{r, s}} \sum_{(k, l) \in I_{r, s},\left(\left\|\frac{\Delta^{v} x_{k, l}-L}{\rho}, z_{1}, \cdots, z_{n-1}\right\|\right) \geq \epsilon} \min \left(\left[M_{k, l}(\epsilon)\right]^{h},\left[M_{k, l}(\epsilon)\right]^{H}\right) \\
& \geq \frac{1}{h_{r, s}}\left|\left\{(k, l) \in I_{r, s}:\left(\left\|\left(\Delta^{v} x_{k, l}-L\right), z_{1}, \cdots, z_{n-1}\right\|\right) \geq \epsilon\right\}\right| \\
& \\
& \min \left(\left[M_{k, l}(\epsilon)\right]^{h},\left[M_{k, l}(\epsilon)\right]^{H}\right) .
\end{aligned}
$$

Hence $x=\left(x_{k, l}\right) \in S_{\theta_{r, s}}\left(\Delta^{v}\right)$.

Theorem 12. Let $\mathcal{M}=\left(M_{k, l}\right)$ be a bounded sequence of Orlicz functions and $0<$ $h=\inf _{k, l} p_{k, l} \leq p_{k, l} \leq \sup _{k, l} p_{k, l}=H<\infty$. Then $S_{\theta_{r, s}}\left(\Delta^{v}\right)$ $\subset\left[A C_{\theta_{r, s}}, \mathcal{M}, \Delta^{v}, p,\|\cdot, \cdots, \cdot\|\right]$.

Proof. Since $M=\left(M_{k, l}\right)$ is bounded, so there exists an integer $K$ such that $M_{k, l}(t)<K$, for all $t \geq 0$. Then

$$
\begin{aligned}
& \frac{1}{h_{r, s}} \sum_{(k, l) \in I_{r, s}}\left[M_{k, l}\left(\left\|\frac{\Delta^{v} x_{k, l}-L}{\rho}, z_{1}, \cdots, z_{n-1}\right\|\right)\right]^{p_{k, l}} \\
& =\frac{1}{h_{r, s}} \sum_{(k, l) \in I_{r, s},\left(\left\|\frac{\Delta^{v} x_{k, l}-L}{\rho}, z_{1}, \cdots, z_{n-1}\right\|\right) \geq \epsilon}\left[M_{k, l}\left(\left\|\frac{\Delta^{v} x_{k, l}-L}{\rho}, z_{1}, \cdots, z_{n-1}\right\|\right)\right]^{p_{k, l}}
\end{aligned}
$$




$$
\begin{aligned}
& +\frac{1}{h_{r, s}} \sum_{(k, l) \in I_{r, s},\left(\left\|\frac{\Delta^{v} x_{k, l}-L}{\rho}, z_{1}, \cdots, z_{n-1}\right\|\right)<\epsilon}\left[M_{k, l}\left(\left\|\frac{\Delta^{v} x_{k, l}-L}{\rho}, z_{1}, \cdots, z_{n-1}\right\|\right)\right]^{p_{k, l}} \\
& \leq \frac{1}{h_{r, s}} \max \left(K^{h}, K^{H}\right) \\
& \quad+\frac{1}{h_{r, s}} \sum_{(k, l) \in I_{r, s},\left(\left\|\frac{\Delta^{v} x_{k, l}-L}{\rho}, z_{1}, \cdots, z_{n-1}\right\|\right) \geq \epsilon} \quad\left[M_{k, l}(\epsilon)\right]^{p_{k, l}} \\
& \leq \max \left(K^{h}, K^{H}\right) \frac{1}{h_{r, s}} \mid\left\{(k, l) \in I_{r, s}:\left(\left\|\frac{\Delta^{v} x_{k, l}-L}{\rho}, z_{x_{k, l}-L}, \cdots, z_{n-1}, \cdots, z_{n-1}\right\|\right)<\epsilon\right. \\
& \quad+\max \left(\left[M_{k, l}(\epsilon)\right]^{h},\left[M_{k, l}(\epsilon)\right]^{H}\right) . \\
& \text { Hence } x=\left(x_{k, l}\right) \in\left[A C_{\theta_{r, s}}, \mathcal{M}, \Delta^{v}, p,\|\cdot, \cdots, \cdot\|\right] .
\end{aligned}
$$

\section{REFERENCES}

[1] A. Alotaibi, M. Mursaleen, and M. Alghamdi, "Invariant and absolute invariant means of double sequences," Jour. Funct. Spaces Appl., vol. 2012, pp. 9 pages, doi:10.1155/2012/465 364., Article ID 465364 .

[2] B. Altay and F. Başar, "Some new spaces of double sequences," Jour. Math. Anal. Appl., vol. 309, pp. 70-90, 2005.

[3] F. Başar and Y. Sever, "The space $\mathscr{L}_{p}$ of double sequences," Math. J. Okayama Univ., vol. 51, pp. 149-157, 2009.

[4] M. Başarir and O. Sonalcan, "On some double sequence spaces," Jour. Indian Acad. Math., vol. 21, pp. 193-200, 1999.

[5] T. J. Bromwich., An introduction to the theory of infinite series. New York: Macmillan and Co. Ltd., 1965.

[6] J. S. Connor, "The statistical and strong p-Cesàro convergence of sequeces," Analysis (Munich), vol. 8, pp. 47-63, 1988.

[7] M. Et and R. Çolak, "On generalized difference sequence spaces," Soochow J. Math., vol. 21, pp. 377-386, 1995.

[8] H. Fast, "Sur la convergence statistique," Colloq. Math., vol. 2, pp. 241-244, 1951.

[9] J. A. Fridy, "On the statistical convergence," Analysis (Munich), vol. 5, pp. 301-303, 1985.

[10] S. Gahler, "Linear 2-normietre Rume," Math. Nachr., vol. 28, pp. 1-43, 1965.

[11] H. Gunawan, "On $n$-inner product, $n$-norms, and the Cauchy-Schwartz inequality," Scientiae Mathematicae Japonicae, vol. 5, pp. 47-54, 2001.

[12] H. Gunawan, "The space of $p$-summable sequence and its natural $n$-norm," Bull. Aust. Math. Soc., vol. 64, pp. 137-147, 2001.

[13] H. Gunawan and M. Mashadi, "On n-normed spaces," Int. J. Math. Math. Sci., vol. 27, pp. 631639, 2001.

[14] G. H. Hardy, "On the convergence of certain multiple series," Proc. Camb. Phil., Soc., vol. 19, pp. 86-95, 1917. 
[15] M. Iş1k, "On statistical convergence of generalized difference sequence spaces," Soochow J. Math., vol. 30, pp. 197-205, 2004.

[16] M. Işı1k, "On statistical convergence of generalized difference sequence spaces," Soochow J. Math., vol. 30, pp. 197-205, 2004.

[17] H. Kızmaz, "On certain sequence spaces," Canad. Math. Bull., vol. 24, pp. 169-176, 1981.

[18] E. Kolk, "The statistical convergence in Banach spaces," Acta. Comment. Univ. Tartu, vol. 928, pp. 41-52, 1991

[19] I. J. Maddox, "A new type of convergence," Math. Proc. Camb. Phil. Soc., vol. 83, pp. 61-64, 1978.

[20] A. Misiak, " $n$-inner product spaces," Math. Nachr., vol. 140, pp. 299-319, 1989.

[21] F. Moricz, "Extension of the spaces $c$ and $c_{0}$ from single to double sequences," Acta Math. Hungarica, vol. 57, pp. 129-136, 1991.

[22] F. Moricz and B. E. Rhoades, "Almost convergence of double sequences and strong reqularity of summability matrices," Math. Proc. Camb. Phil. Soc., vol. 104, pp. 283-294, 1988.

[23] M. Mursaleen, "Generalized spaces of difference sequences," Jour. Math. Analy. Appl., vol. 203, pp. 738-745, 1996.

[24] M. Mursaleen, "Almost strongly regular matrices and a core theorem for double sequences," Jour. Math. Anal. Appl., vol. 293, no. 2, pp. 523-531, 2004.

[25] M. Mursaleen, A. Alotaibi, and S. Mohiuddine, "Statistical convergence through de la ValléePoussin mean in locally solid Riesz spaces," Adv. Diff. Equ., vol. 2013, pp. 66, doi:10.1186/16871847-2013-66., 2013.

[26] M. Mursaleen, C. Çakan, S. A. Mohiuddine, and E. Savaş, "Generalized statistical convergence and statitical core of double sequences," Acta Math. Sinica, vol. 26, no. 11, pp. 2131-2144, 2010.

[27] M. Mursaleen and O. H. H. Edely, "Statistical convergence of double sequences," Jour. Math. Anal. Appl., vol. 288, no. 1, pp. 223-231, 2003.

[28] M. Mursaleen and O. H. H. Edely, "Almost convergence and a core theorem for double sequences," Jour. Math. Anal. Appl., vol. 293, no. 2, pp. 532-540, 2004.

[29] M. Mursaleen and S. A. Mohiuddine, "Double $\sigma$-multiplicative matrices," J. Math. Anal. Appl., vol. 327, pp. 991-996, 2007.

[30] M. Mursaleen and S. A. Mohiuddine, "Almost bounded variation of double sequences and some four dimensional summability matrices," Publ. Math. Debrecen, vol. 75, pp. 495-508, 2009.

[31] M. Mursaleen and S. A. Mohiuddine, "Statistical convergence of double sequences in intuitionistic fuzzy normed spaces," Chaos Solitons Fractals, vol. 41, pp. 2414-2421, 2009.

[32] T. Salat, "On statictical convergent sequences of real numbers," Math. Slovaca, vol. 30, pp. 139_ $150,1980$.

[33] E. Savaş, "Some new double sequence spaces defined by Orlicz function in $n$-normed space," Jour. Ineq. Appl., vol. ID 592840, pp. 1-9, 2011.

[34] I. J. Schoenberg, "The integrability of certain functions and related summability methods," Amer. Math. Monthly, vol. 66, pp. 361-375, 1969.

Authors' addresses

\section{Mursaleen}

Department of Mathematics,, Aligarh Muslim University, Aligarh-202002, India

E-mail address: mursaleenmegmail.com

\section{Kuldip Raj}

School of Mathematics,, Shri Mata Vaishno Devi University, Katra - 182320, J\&K, INDIA

E-mail address: kuldipraj68@gmail.com 
Sunil K. Sharma

Department of mathematics, Model Institute of Engineering \& Technology, Kot Bhalwal-181122, $\mathrm{J} \& \mathrm{~K}$, INDIA

E-mail address: sunilksharma42@gmail.com 